\title{
Formación profesional y género: desigualdades que persisten, algunos cambios y debates pendientes
}

\author{
Rafael Merino'
}

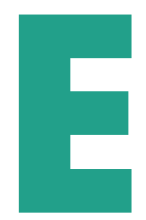

n el año 2015 se publicó un monográfico en la revista International Journal of Vocational Training que, de hecho, era una réplica de un primer monográfico que ya se publicó en el año 2006. Entre las razones que justificaba la aparición de un segundo monográfico era la persistente desigualdad de género en la formación profesional. De todo el sistema educativo es en la formación profesional donde se mantienen las mayores desigualdades por razón de género. Una segunda razón era que en los casi 10 años después del primer monográfico no llegaba al 4\% los artículos publicados con perspectiva substantiva de género (Niemeyer y Colley, 2015). No hay métrica para revistas en lengua española, pero es razonable pensar que el panorama no sea mucho mejor. Por eso parece del todo pertinente la aparición en lengua española de este monográfico que da cuenta de investigaciones actuales en el campo de la sociología de la educación, investigaciones realizadas en España y en Argentina, con perspectivas cuantitativas y cualitativas, y con numerosos estudios de caso que tienen en cuenta el contexto territorial en el marco de análisis.

El monográfico «Formación Profesional y Género» incluye un primer artículo que firman Aguado, Cano y Sánchez donde se constata la conocida feminización y masculinización de las familias profesionales de la formación profesional (FP), aunque también se constatan algunos pequeños cambios como el aumento de hombres en ciclos feminizados, pero no a la inversa. La diferencia por género de las familias profesionales también es objeto de análisis del segundo artículo, de Ortega, Piciucchi, Ruiz-Callado y de Gracia Soriano, con la introducción del papel de la orientación en las elecciones escolares y con un innovador enfoque que integra el análisis de la FP dentro de un plan local de juventud. El artículo de Cora y Barbetti analiza los mecanismos de legitimación de las diferentes elecciones y expectativas de chicas y chicos en relación a la formación profesional, en un contexto de fuertes movilizaciones feministas en los últimos años como es el caso de Argentina. El artículo de Obiol-Francés, Almeda, Di Nella, Pumar, Ruiz, Vegés-Bosch y Villar-Aguilés pone el foco en el nivel básico de FP, donde la segregación por género es más acusada, y en parte se explica por la prioridad de evitar el abandono de la formación, lo que plantea un dilema a las políticas públicas. El artículo de Vidal y Merino analiza las motivaciones de jóvenes, chicos y chicas, para escoger formación profesional, motivaciones en las que se cruzan las identidades de género con el cálculo en el retorno educativo y laboral de la formación, y también se analiza el sesgo de género en el abandono de la formación. El artículo de Dionisio y Lewin introduce de forma explícita la perspectiva interseccional para el análisis de las desigualdades que afectan a las mujeres, con el cruce con la etnia y el

1 Grupo de Investigación Educación y Trabajo; Departamento de Sociología, Universidad Autónoma de Barcelona. 
nivel socioeconómico, en una ciudad mediana de Argentina. La aportación de Jacinto, Millenaar, Roberti, Burgos y Sosa se centra en el análisis de la incorporación de mujeres en un ámbito profesional masculinizado como la programación informática, donde se vislumbran las dificultades de esta incorporación, pero también las estrategias que pueden superar los estereotipos de género. Para acabar el monográfico, el artículo de Martínez, Martín, Garino, Giampaoletti y Pol plantean diferencias entre los discursos de jóvenes y de directivos de centros de formación en dos contextos socioeconómicos diferenciados, dos provincias de Argentina con base económica muy diferente, que incide en la naturalización o la visibilización de las jerarquías de género.

Este conjunto de artículos arroja algunas evidencias y aporta elementos para una agenda futura de investigación en formación profesional y género. Las evidencias se pueden resumir en tres: la persistente desigualdad entre modalidades profesionales feminizadas y masculinizadas; la dificultad de adentrarse en campo contrario, especialmente para las chicas entrar en ámbitos profesionales dominados por chicos, y especialmente en los niveles básicos de formación; y la persistente segregación horizontal en el mercado de trabajo. Una cuestión más compleja es la búsqueda de mecanismos que contribuyan a explicar la persistencia de estas desigualdades. El principal mecanismo es la socialización diferencial, anclada en patrones tradicionales de división sexual del trabajo y generadora de identidades sexuales y de género que condicionan las elecciones de chicas y chicos. Una cuestión de fondo ciertamente polémica es cuando disminuye esta socialización diferencial en la familia y en la escuela obligatoria, pero en la transición a la educación post-obligatoria continúan manteniéndose las elecciones diferentes por parte de chicas y chicos, lo que se ha llamado la paradoja nórdica de la igualdad, porque se ve claramente en los países nórdicos, o en general paradoja de la igualdad de género (que ya tiene entrada en Wikipedia). No es este el espacio para abordar esta paradoja, pero sí que es un desafío para la perspectiva culturalista dominante en la sociología en general, y en los estudios de género en particular.

Pero, además, la socialización es imperfecta. La posibilidad de cambio, o de «transfuguismo» o de «transclasismo» (Jaquet, 2014) surge cuando esta socialización deja algunas grietas por las que se pueden colar elecciones no esperadas, dando lugar a «transidentidades» (Alessandrin et al., 2014). Las reacciones resocializadoras han perdido la fuerza de antaño, en un contexto de movilizaciones feministas en pro de la igualdad. Como muestran algunos artículos del monográfico, al nivel de discurso las prácticas discriminadoras están mal valoradas. Hay un discurso por lo general igualitario y de rechazo a los argumentos tradicionales de la división sexual del trabajo que encasilla a las chicas en ramas profesionales «femeninas» y que denostaba las chicas que osaban entrar en territorio «masculino». Otra cuestión es que se puedan ejercer estas prácticas de forma más sutil, con mecanismos más complejos como la hipervisualización de las chicas que entran en campos formativos de mecánica o similares (Lamamra, 2017).

También hay que poner en contexto la relación entre el sistema educativo y el mercado de trabajo. Un sistema educativo con una diferenciación curricular temprana y con vías profesionalizadoras especializadas favorece la elección tradicional, en cambio una mayor comprensividad y una menor especialización desincentiva el marcador de género en las elecciones (Imdorf et al., 2015). El mercado de trabajo, con menos presión a políticas igualitarias que el sistema educativo, ofrece un campo restringido de oportunidades, con lo que una elección educativa tradicional puede contemplarse como una estrategia racional en función a las oportunidades reales y percibidas por chicos y chicas (Martínez García, 2017). 
Aunque el enfoque del monográfico es más analítico que propositivo, de los artículos se pueden deducir algunos retos para las políticas educativas, como el tema clave de la orientación en la secundaria y el tema de la oferta formativa más o menos genérica. En la orientación se puede plantear la modificación de preferencias de chicas y chicos, para evitar que los patrones sexistas se reproduzcan, y en las políticas de oferta se pueden plantear cursos de ámbitos tecnoindustriales solo para chicas, en los que se puedan combinar identidades femeninas con trabajos tradicionalmente atribuidos a hombres, como se puede apreciar en el original y premiado documental Ouaga Girls², que muestra un grupo de chicas estudiando mecánica en una escuela de la capital de Burkina Fasso. También se puede ver en el documental que el problema fundamental está en encontrar centros de trabajo para hacer prácticas y para la inserción laboral, menos en una empresa que está dirigida por una mujer.

Pero si las prácticas de orientación y las políticas de oferta se reducen a la promoción de chicas en modalidades tecnológicas o industriales, aparece un efecto perverso porque se profundiza la desvalorización de las modalidades feminizadas. Estas modalidades también pueden ser un espacio de empoderamiento y, no hay que olvidar, de mayores oportunidades laborales, sobre todo en los niveles menos cualificados de formación. Sea en la estética o en la sanidad, las familias feminizadas de la formación profesional pueden y deben estar en el centro de las políticas a desarrollar en el futuro próximo, como está dejando claro la pandemia que nos asola a toda la humanidad. Si es que aprendemos algo de la experiencia, estos ámbitos formativos-laborales serán uno de los pilares para la transición hacia la economía de los cuidados (Duran, 2018).

\section{Referencias bibliográficas}

Alessandrin, Arnaud; Espineira, Karine y Thomas, Maud-Yeuser (2014). Tableau noir: les transidentités et l'école. Paris: Éditions L'Harmattan.

Niemeyer, Beatrix y Colley, Helen (2015): "Why Do We Need (Another) Special Issue on Gender and VET?”. Journal of Vocational Education \& Training, 67 (1), 1-10, DOI: http://dx.doi.org/10.1080/1363 6820.2014.971498.

Duran, M. Angeles (2018). La riqueza invisible del cuidado. Valencia: Universitat de València, Servei de Publicacions.

Imdorf, Christian; Hegna, Kristinn y Reisel, Liza (2015). Gender Segregation in Vocational Education. United Kingdom: Emerald Group Publishing Limited.

Jaquet, Chantal (2014). Les Transclasses ou la non-reproduction. Paris: PUF.

Lamamra, Nadia (2017): "Vocational Education and Training in Switzerland: A gender Perspective. From Socialisation to Resistance”. Educar, 53 (2), 379-396. DOI: http://dx.doi.org/10.5565/rev/educar.863.

Martínez García, José Saturnino (2017). La equidad y la educación. Madrid: Los Libros de la Catarata.

.2 https://eldocumentaldelmes.com/es/doc/ouaga-girls-2/ 